\title{
Incidencia de diabetes mellitus tipo 1 en Santiago de Chile: análisis por comunas de la Región Metropolitana en el período 2000-2004
}

\author{
Elena Carrasco Pla, Bárbara Angel $B^{2 b}$, Ethel Codner ${ }^{3}$, \\ Diego García D ${ }^{1 c}$, Francisca U garte $P^{4}$, M Eugenia \\ Bruzzone V5, Francisco Pérez $B^{2 d}$. \\ Type 1 diabetes mellitus incidence \\ in Santiago, Chile. Analysis by \\ counties in the period 2000-2004
}

Background: There are great geographical differences in the incidence of type I diabetes mellitus. Aim: To determine the incidence rate of type 1 diabetes mellitus (DM1) in the Metropolitan Region of Santiago, Chile from January 1, 2000 to December 31, 2004 and to observe the distribution of cases in the different counties of Santiago. Material and methods: All the cases diagnosed with DM1 in the Metropolitan Region who fulfilled the following requirements were included in the study: age of onset $<15$ years, insulin treatment from onset, permanent residency in the area, and a diagnosis made between January, 2000 and December, 2004. Results: The incidence of DM1 was 6.58/ 100.000 inhabitants/year, and showed a significant increase from 2001 to 2004 (5.44 and 8.33 inhabitants/year, respectively, $\mathrm{p}<0.04$ ). The incidence of DM1 also increased significantly in children younger than 4 years old. The incidence by counties exhibited large differences, ranging from 1,5 to 26,6/100.000 inhabitants. Counties with higher income, urbanization and low aborigine component showed a high incidence rate of type 1 diabetes. Conclusions: In the Metropolitan Region of Santiago, an increase of the incidence of DM1 has occurred in the period 2000-2004, especially in children younger than 4 years old. Large differences among counties were observed (Rev Méd Chile 2006; 134: 1258-64).

(Key words: Diabetes Mellitus, juvenile-onset; Diabetes Mellitus, type 1; Epidemiology)

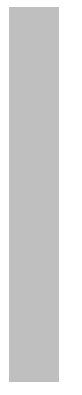
Recibido el 15 de julio, 2005. Aceptado el 7 de abril, 2006
aNutricionista, MSc
bMatrona, MSc, Candidata a Doctor en Nutrición
cBiotecnólogo
dDoctor en Ciencias Biológicas

${ }^{1}$ Unidad de Diabetes, Hospital San Juan de Dios, Departamento de Medicina Occidente, Facultad de Medicina, Universidad de Chile. ${ }^{2}$ Laboratorio de Investigación en Epidemiología Nutricional y Genética, INTA, Universidad de Chile. ${ }^{3}$ Instituto de Investigaciones Materno Infantil (IDIMI), Facultad de Medicina, Universidad de Chile y Hospital Clínico San Borja Arriarán. ${ }^{4}$ Unidad de Endocrinología, Hospital Exequiel González Cortés. ${ }^{5}$ Departamento de Fisiología, ICBM, Facultad de Medicina, Universidad de Chile.

Correspondencia a: Sra. Elena Carrasco P. Unidad de Diabetes, Hospital San Juan de Dios. Santiago de Chile. Fax: 56-2-6825465. E mail: ecarrasc@med.uchile.cl 
L a diabetes mellitus tipo 1 se caracteriza por la destrucción autoinmune de las células beta secretoras de insulina localizadas en el páncreas ${ }^{1}$. En los últimos años, la metodología proporcionada por la OMS y el Proyecto DIAMOND (Diabetes Mondiale) ha confirmado, definitivamente, la existencia de grandes diferencias geográficas en las tasas de incidencia de la diabetes mellitus tipo 1 alrededor del mundo ${ }^{2}$. Esta gran variación sugiere que en la etiología de la diabetes mellitus tipo 1 participan, en el proceso de destrucción de la célula beta, tanto factores genéticos como ambientales $^{3}$. Las tasas más altas de incidencia en el mundo corresponden a los países del norte de Europa, tales como Noruega, Finlandia, Dinamarca y en la isla de Cerdeña, en Italia ${ }^{4}$. Por otra parte, tasas muy bajas fueron determinadas en países asiáticos, así como en regiones de China y Corea ${ }^{5,6}$.

La población chilena está constituida por una mezcla de diferentes grupos étnicos, incluyendo población europea (predominantemente de España) $\mathrm{y}$ amerindios (principalmente mapuches y aymaras) ${ }^{7}$. Según el censo de población del año 2002, los nativos corresponden a $10 \%$ de la población total ${ }^{8}$. La diabetes mellitus tipo 1 en población mapuche ha sido descrita como un evento excepcionalmente esporádico y casi inexistente en menores de 15 años. Larenas y cols ${ }^{9}$ reportaron una incidencia de 0,43/ 100.000 por año en este grupo étnico, mientras que la tasa de incidencia de la Región Metropolitana, en este mismo grupo de edad, fue estimada en 2,36/ 100.000 por año en el período 1986-1992 ${ }^{10}$. Dado que el componente amerindio se distribuye mayoritariamente en ciertas comunas de la Región Metropolitana, los objetivos de este estudio fueron determinar la tasa de incidencia de diabetes mellitus tipo 1 en menores de 15 años, en la Región Metropolitana entre el $1^{0}$ de enero del año 2000 hasta el 31 de diciembre de 2004 y observar las diferencias por comunas.

\section{MÉTodos}

Área de estudio. La Región Metropolitana concentra $40 \%$ del total de la población chilena. De acuerdo al censo del año $2002^{8}$, la población total alcanzó la cifra de 6.061.185 habitantes, de esta población, 96,6\% vive en condiciones urbanas. La población menor de 15 años de la Región Metro- politana es de 1.509 .218 niños. Las comunas con mayor número de habitantes corresponden a: Maipú (468.390), La Florida (365.674), Las Condes (249.893), Peñalolén (216.060) y Santiago (200.792).

Criterios de inclusión. Para ser incluido en el registro de incidencia se consideró:

1. Diagnóstico de diabetes mellitus tipo 1 desde el comienzo.

2. Edad menor de 15 años.

3. Uso de insulina desde el inicio.

4. Residencia en la Región Metropolitana, comunas urbanas y rurales.

Además, se estableció como fecha de diagnóstico el día de la primera inyección de insulina. Como fuente primaria, se utilizaron los registros de los servicios de urgencia pediátrico y de adulto (hasta 14 años, 11 meses y 29 días), las fichas clínicas de todos los hospitales, estatales, privados y de las fuerzas armadas, y como fuente secundaria independiente, la información de la Fundación de Diabetes Juvenil de Chile. Debido a la severidad de la enfermedad y al costo del tratamiento la mayoría de los diabéticos tipo 1 se encuentran adscritos a algún sistema de salud, público 0 privado (Programa AUGE). Es difícil por lo tanto, que algún niño con esta enfermedad no esté registrado en algún centro de salud. Es por eso que la concordancia de las fuentes (ascertainment) es cercana a $100 \%$, es decir, que el caso nuevo esté registrado, a lo menos, en una fuente de información. Se utilizó el método de captura y recaptura ${ }^{11}$.

Estadística. Para cada año, la población menor de 15 años obtenida desde las proyecciones postcensales, fue utilizada como denominador en la tasa de incidencia. El número de casos recién diagnosticados se empleó como numerador. La incidencia se expresó como el número de casos por 100.000 habitantes menores de 15 años por año. El intervalo de confianza a 95\% fue calculado de acuerdo a la distribución de Poisson. Considerando la población mundial ${ }^{12}$ y tomando el período total 2000-2004, se realizó análisis estratificado con el objetivo de tabular la tasa de incidencia según sexo y grupo de edad. Todos los análisis estadísticos fueron realizados usando el programa estadístico STATA 8.2. 
Tabla 1. Tasas anuales de incidencia/ 100.000 , en menores de 15 años por sexo y por año

\begin{tabular}{|c|c|c|c|c|c|c|c|c|}
\hline \multirow[t]{2}{*}{ Años } & \multicolumn{3}{|c|}{ Niños } & \multicolumn{3}{|c|}{ Niñas } & \multicolumn{2}{|c|}{ Total } \\
\hline & $\mathrm{n}$ & Inc. & (IC 95\%) & $\mathrm{n}$ & Inc. & (IC 95\%) & Inc. & (IC 95\%) \\
\hline 2000 & 39 & 5,08 & $(3,23-6,11)$ & 43 & 5,80 & $(3,25-6,19)$ & 5,44 & $(4,33-6,76)$ \\
\hline 2001 & 53 & 6,90 & $(3,77-7,46)$ & 38 & 5,13 & $(3,17-5,98)$ & 6,02 & $(4,86-7,42)$ \\
\hline 2002 & 49 & 6,38 & $(3,13-7,34)$ & 35 & 4,72 & $(3,30-6,19)$ & 5,55 & $(4,45-6,90)$ \\
\hline 2003 & 65 & 8,47 & $(4,03-9,12)$ & 49 & 6,61 & $(3,15-7,66)$ & 7,54 & $(6,24-9,09)$ \\
\hline 2004 & 61 & 7,47 & $(5,74-9,78)$ & 68 & 9,19 & $(7,14-11,64)$ & 8,33 & $(7,15-11,02)$ \\
\hline Total & 267 & 7,16 & $(6,08-7,78)$ & 233 & 6,08 & $(5,51-7,16)$ & 6,58 & $(6,03-7,21)$ \\
\hline
\end{tabular}

\section{Resultados}

Los datos de incidencia anual según sexo se muestran en la Tabla 1. Se encontraron 500 niños con diabetes mellitus tipo 1 menores de 15 años en el período estudiado (267 niños y 233 niñas). La tasa promedio de incidencia determinada para el período total 2000-2004 fue de 6,58/100.000 hab/año. La tasa de incidencia registrada en el año 2000 (5,44/100.000 hab/año) tuvo un incremento significativo hasta llegar al año 2004 con una tasa de 8,33/100.000 hab/año ( $p<0,04)$.

La Figura 1 resume el número de casos por grupos de edad y por sexo en el período analizado. Al realizar el análisis estadístico, de acuerdo a la distribución de Poisson, éste indicó que el riesgo de encontrar diabéticos en el grupo de 5-9 años fue de $1,67(1,31-2,12)$ y de $1,58(1,25-$ 2,00 ) en el grupo de 10-14 años respecto al grupo de 0-4 años ( $\mathrm{p}<0,001)$. Al realizar el análisis estratificado según sexo (riesgo mujeres/riesgo hombres) se obtuvo un valor de 0,88 $(0,74-1,06)$ ( $p=0,71$ ), es decir, la enfermedad en este período es más frecuente en hombres, sin embargo, dado el valor de $\mathrm{p}$, no hay interacción entre la variable sexo y el mayor riesgo de desarrollar diabetes tipo 1. Cabe destacar el notable incremento que ha experimentado la diabetes mellitus tipo 1 en el grupo de niños de 0-2 años, registrándose un total de 46 niños distribuidos de la siguiente forma: año 2000 (2 casos); año 2001 (6 casos); año 2002 (8 casos); años 2003 y 2004 (15 casos, respectivamente). Este aumento también se refleja en el incremento de la tasa de incidencia del grupo de 0-4 años en el año 2000 (3,54/100.000 hab/año), cifra que se duplicó en el año $2004(7,30 / 100.000$ hab/año) $(p<0,01)$.
En relación a la estacionalidad, el número total de casos diagnosticados fue significativamente mayor durante el período otoño-invierno comparado con primavera-verano (267 casos versus 233 casos, $p=0,037)$. Observándose la mayor diferencia en el año 2001 (59 casos versus 32 casos, $\mathrm{p}<0,001$ ).

Finalmente, la Tabla 2 muestra la distribución total de casos diagnosticados en el período 20002004 y la tasa de incidencia cruda de cada comuna de la Región Metropolitana (urbanas y rurales). Dentro de las comunas urbanas, el análisis muestra una mayor tasa de incidencia (/100,000 hab-año) en: Vitacura $(18,87)$, Providencia $(19,59)$, Lo Barnechea $(19,90)$, Las Condes $(13,70)$, La Reina $(13,88)$ y Ñuñoa $(14,45)$. Por otra parte, las cifras de menor incidencia para el período estudiado se observaron

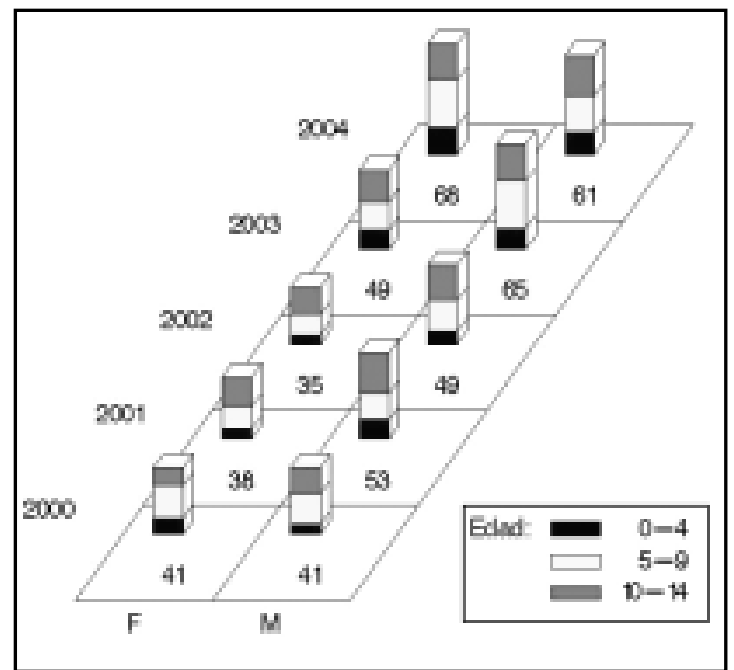

FIgURA 1. Número de casos diagnosticados en el Gran Santiago de acuerdo a grupos de edad y sexo en el período 2000-2004. 
Tabla 2. N úmero de casos debutantes y tasa de incidencia (100,000 hab. año) por comuna (urbana y rural) para el período 2000-2004

\begin{tabular}{|c|c|c|c|c|c|c|c|c|}
\hline \multicolumn{2}{|c|}{ Comuna (no hab) Años } & 2000 & 2001 & 2002 & 2003 & 2004 & Total & $\begin{array}{l}\text { Tasa/ } \\
100,000\end{array}$ \\
\hline \multirow[t]{34}{*}{ URBANAS } & Cerrillos (17.511) & 2 & 0 & 1 & 1 & 1 & 5 & 5,71 \\
\hline & Cerro Navia (39.511) & 0 & 2 & 3 & 0 & 4 & 9 & 4,56 \\
\hline & Conchalí (30.885) & 2 & 0 & 1 & 2 & 3 & 8 & 5,18 \\
\hline & El Bosque (45.311) & 3 & 3 & 1 & 2 & 2 & 11 & 4,86 \\
\hline & Estación Central (28.140) & 2 & 1 & 1 & 2 & 0 & 6 & 4,26 \\
\hline & Huechuraba (20.765) & 1 & 4 & 1 & 2 & 0 & 8 & 7,70 \\
\hline & Independencia (12.571) & 4 & 0 & 2 & 0 & 1 & 7 & 11,14 \\
\hline & La Cisterna (18.437) & 1 & 1 & 1 & 0 & 1 & 4 & 4,34 \\
\hline & La Florida (87.425) & 8 & 8 & 3 & 7 & 8 & 34 & 7,78 \\
\hline & La Granja (34.342) & 1 & 2 & 2 & 2 & 1 & 8 & 4,66 \\
\hline & La Pintana (55.350) & 1 & 2 & 1 & 5 & 3 & 12 & 4,34 \\
\hline & La Reina (21.617) & 2 & 1 & 4 & 5 & 3 & 15 & 13,88 \\
\hline & Las Condes (48.138) & 6 & 7 & 10 & 5 & 5 & 33 & 13,70 \\
\hline & Lo Barnechea (21.097) & 2 & 3 & 2 & 8 & 6 & 21 & 19,90 \\
\hline & Lo Espejo (30.115) & 0 & 1 & 0 & 3 & 0 & 4 & 2,66 \\
\hline & Lo Prado (24.777) & 3 & 0 & 2 & 3 & 1 & 9 & 7,26 \\
\hline & Macul (24.213) & 3 & 1 & 3 & 1 & 5 & 13 & 10,74 \\
\hline & Maipú (128.408) & 6 & 4 & 5 & 7 & 16 & 38 & 5,92 \\
\hline & Nuñ̃oa (29.059) & 5 & 4 & 3 & 6 & 3 & 21 & 14,45 \\
\hline & Pedro Aguirre Cerda (26.200) & 0 & 1 & 1 & 1 & 0 & 3 & 2,29 \\
\hline & Peñalolén (58.889) & 1 & 4 & 3 & 3 & 10 & 21 & 7,13 \\
\hline & Providencia (16.339) & 5 & 1 & 1 & 5 & 4 & 16 & 19,59 \\
\hline & Pudahuel (54.973) & 0 & 3 & 1 & 1 & 4 & 9 & 3,27 \\
\hline & Puente Alto (144.207) & 6 & 12 & 9 & 6 & 12 & 45 & 6,24 \\
\hline & Quilicura (39.377) & 1 & 1 & 1 & 0 & 1 & 4 & 2,03 \\
\hline & Quinta Normal (22.964) & 0 & 1 & 0 & 5 & 2 & 8 & 6,97 \\
\hline & Recoleta (33.827) & 1 & 1 & 1 & 1 & 5 & 9 & 5,32 \\
\hline & Renca (35.125) & 2 & 1 & 1 & 3 & 2 & 9 & 5,12 \\
\hline & San Bernardo (69.473) & 3 & 3 & 3 & 8 & 6 & 23 & 6,62 \\
\hline & San Joaquín (21.046) & 1 & 0 & 2 & 2 & 0 & 5 & 4,75 \\
\hline & San Miguel (15.687) & 1 & 0 & 3 & 0 & 2 & 6 & 7,65 \\
\hline & San Ramón (24.233) & 0 & 1 & 2 & 2 & 2 & 7 & 5,78 \\
\hline & Santiago (31.279) & 1 & 3 & 3 & 3 & 3 & 13 & 8,31 \\
\hline & Vitacura (15.902) & 4 & 3 & 3 & 3 & 2 & 15 & 18,87 \\
\hline \multirow[t]{13}{*}{ RURALES } & El Monte (7.444) & 0 & 0 & 0 & 1 & 0 & 1 & 2,69 \\
\hline & Isla de Maipo (7.025) & 0 & 0 & 2 & 0 & 1 & 3 & 8,54 \\
\hline & Tiltil (4.365) & 0 & 1 & 0 & 0 & 0 & 1 & 4,58 \\
\hline & Lampa (12.387) & 0 & 1 & 0 & 0 & 2 & 3 & 4,84 \\
\hline & Colina (22.184) & 0 & 1 & 0 & 3 & 3 & 7 & 6,31 \\
\hline & Pirque (4.758) & 0 & 1 & 0 & 0 & 0 & 1 & 4,20 \\
\hline & Calera de Tango (5.350) & 1 & 0 & 0 & 0 & 1 & 2 & 7,49 \\
\hline & Paine (14.016) & 0 & 1 & 0 & 1 & 2 & 4 & 5,70 \\
\hline & Curacaví (6.566) & 0 & 1 & 1 & 0 & 0 & 2 & 6,09 \\
\hline & Melipilla (25.409) & 1 & 1 & 0 & 1 & 2 & 5 & 3,94 \\
\hline & Padre Hurtado (10.584) & 1 & 3 & 0 & 4 & 1 & 9 & 17,00 \\
\hline & Peñaflor (18.280) & 1 & 0 & 0 & 0 & 0 & 1 & 1,09 \\
\hline & Talagante (17.210) & 0 & 2 & 0 & 0 & 0 & 2 & 2,32 \\
\hline
\end{tabular}

San José de Maipo (3.291 hab), Buin (17.230 hab), Alhué (1.211 hab), María Pinto (2.807 hab) y San Pedro (1.908 hab) no registraron casos de diabetes tipo 1 durante el período analizado. 
en las comunas de: Pedro Aguirre Cerda $(2,29)$, Lo Espejo $(2,66)$ y Quilicura $(2,03)$. En cuanto a las comunas rurales, la de mayor incidencia fue: Padre Hurtado $(17,00)$ e Isla de Maipo $(8,54)$. No se registraron casos nuevos para este período en las comunas de: San José de Maipo, Buin, Alhué, María Pinto y San Pedro. Debemos señalar que el cálculo de la tasa de incidencia presentado en la Tabla 2, corresponde a un análisis crudo. Una forma de suavizar las estimaciones sería utilizando el algoritmo de Head-banging (http://srab.cancer.gov/headbang/) que usa la información de las comunas vecinas para estabilizar las tasas, especialmente en aquellas comunas menos pobladas.

\section{DisCUSIÓN}

La diabetes mellitus tipo 1 resulta principalmente de la destrucción autoinmune de las células beta del páncreas. Las causas que desencadenan este proceso destructivo no se conocen exactamente, pero se sabe que involucran factores genéticos y no genéti$\cos ^{1,3}$. Algunos elementos del medio ambiente, como virus o factores nutricionales, también podrían jugar un papel importante, sobre todo en las primeras etapas de la vida, especialmente a nivel intrauterino ${ }^{13}$. El incremento en la tasa de incidencia en poblaciones estables desde el punto de vista genético como es la de Chile, hace suponer claramente la influencia de factores ambientales ${ }^{14,15}$.

El aumento de la incidencia de la diabetes tipo 1 es un hecho reconocido en el mundo. El Proyecto DiaMond así lo ha confirmado ${ }^{2}$. Este incremento sostenido en las tasas de incidencia también se ha reflejado en nuestro país. Es posible que durante los primeros años del registro en Chile, pudiera haber existido un subregistro, sin embargo, en la última década la metodología aplicada en la captura de casos nuevos está completamente instaurada y la curva ascendente se ha mantenido con incrementos significativos ${ }^{16}$.

Este aumento tiene algunas características especiales: los países con altas tasas de incidencia como Finlandia y otros países nórdicos, han mantenido su incremento en las tasas en forma menor, sin embargo, aquellos países que tenían tasas de nivel medio o bajo muestran en el último tiempo un incremento mucho más agresivo ${ }^{17-19}$. Particular es el caso de Chile, que hasta hace 10-12 años estaba incorporado en el grupo de países con tasas de incidencia baja y actualmente se ubica entre los países con incidencia intermedia ${ }^{16}$.

Existen varias especulaciones que podrían ser útiles para explicar este rápido aumento. Claramente, la influencia de factores ambientales aun supuestos, podrían ser los responsables de este aumento. La pesquisa de niños diabéticos de menor edad supone la participación de factores tales como: infecciones perinatales, incremento de peso en los primeros meses de vida (se ha indicado que aumenta 1,5 veces el riesgo de desarrollar diabetes, especialmente en las niñas) y aumento de la edad materna ${ }^{13,21-25}$.

Una asociación entre alto peso de nacimiento 0 alto peso de nacimiento para la edad gestacional y la diabetes mellitus tipo 1 ha sido propuesta en diversos estudios. Sin embargo, es una hipótesis que no ha sido confirmada por todos los estu$\operatorname{dios}^{26-28}$. La incidencia de diabetes mellitus tipo 1 aumenta en forma casi lineal con el peso al nacer, es decir que a mayor peso, existe una mayor probabilidad de desarrollar esta enfermedad.

Hasta el momento, todos los resultados sólo permiten especular sobre los mecanismos biológicos que podrían explicar estos resultados. La insulina es el factor de crecimiento fetal más importante en la última etapa del embarazo. Estudios in vitro, han demostrado que las células beta que más activamente segregan insulina, son más susceptibles de ser dañadas por mediadores propios del crecimiento, comparadas con las células beta menos activas ${ }^{3}$. De este modo, un mayor crecimiento intrauterino podría condicionar un riesgo aumentado en la destrucción de las células beta pancreáticas, evento mediado por componentes inmunológicos o apoptóticos ${ }^{29}$.

Investigaciones realizadas en Noruega y otros países indican que la edad de la madre no tiene ningún efecto en el riesgo del primer hijo de desarrollar diabetes mellitus tipo 1, pero sí puede influir sobre el riesgo de desarmollar la enfermedad en los nacidos posteriormente. El estudio noruego publicado en el año 2001, se basa en el rastreo de los 1,4 millones de personas nacidas entre $1974 \mathrm{y}$ 1998 en Noruega. Los investigadores identificaron un total de 1.824 casos de diabetes tipo 1, diagnosticados entre 1989 y 1998. Según reveló el estudio, en el cuarto hijo, el riesgo de diabetes se incrementaría $43,2 \%$ por cada incremento de 5 años en la edad de 
la madre. Los autores también verificaron esta asociación en los niños que nacieron en segundo y en tercer lugar, pero no en los primogénitos ${ }^{30,31}$.

Estos hallazgos podnían interpretarse desde el punto de vista de la participación de ciertos componentes biológicos asociados a la relación madre-hijo. Biológicamente, la interacción entre los sistemas inmunes del feto y de la madre puede variar, ya sea por la edad de la madre o con cada embarazo, incidiendo en el riesgo de diabetes. La alimentación, el cuidado y la exposición del recién nacido a las infecciones puede variar de acuerdo a la edad de la madre y al número de hijos que ésta tiene ${ }^{32}$.

En nuestro estudio se ha observado, aunque no con significancia estadística, un incremento en el número de niños debutantes con edades bajo los 2 años, situación difícil de encontrar con anterioridad.

Otro de los componentes que podrían estar marcando posibles diferencias comunales para la tasa de incidencia, podría ser la composición étnica de la población. Desde esta perspectiva, nuestros datos de mayor incidencia claramente corresponden a aquellas comunas donde el componente indígena es bajo (Providencia 1,2\%; Las Condes 1,3\%, Vitacura 1,7\% y La Reina 1,8\%) ${ }^{33}$.

Del mismo modo, al observar algunos indicadores socio-económicos como el ingreso moneta-

\section{REFERENCIAS}

1. Puglese A. Genetics of type 1 diabetes. Endocrinol Metab Clin N Am 2004; 33: 1-16.

2. World Health Organization DIAMOND Project Group on Epidemics. Childhood diabetes, epidemics, and epidemiology: an approach for controlling diabetes. Am J Epidemiol 1992; 135: 803-16.

3. KaWAsaki E, Abiru N, Eguchi K. Prevention of type 1 diabetes: from the view point of $\beta$ cell damage. Diab Res Clin Pract 2004; 66 (Suppl 1): 27-32.

4. Green A, Gaie EAM, Patterson CC. Incidence of childhood-onset insulin-dependent diabetes mellitus: the Eurodiab Ace Study. Lancet 1992; 339: 905-9.

5. Yang Z, Wang $K, L \mathrm{~T}$, Sun $\mathrm{W}, \mathrm{L} \mathrm{Y}$, Chang YF et aL Childhood diabetes in China. Enormous variation by place and ethnic group. Diabetes Care 1998; 21: 525-9.

6. Ko KW, Yang SW, CHo NH. The incidence of IDDM in Seoul from 1985 to 1988. Diabetes Care 1994; 17: 1473-5. rio, las comunas de mayor ingreso también muestran las mayores tasas de incidencia. Por otro lado, el análisis de indicadores de pobreza y de saneamiento ambiental (hacinamiento y eliminación de excretas) muestran que las comunas más pobres son aquellas que tienen los valores más bajos de incidencia ${ }^{34}$. Lo anterior ya ha sido descrito en el último tiempo y corresponde a una temática muy controversial denominada «eoría de la higiene», que indica que aquellas poblaciones con los mayores índices de urbanización estarían menos expuestos a los antígenos ambientales y, por lo tanto, sus mecanismos de desarrollo de memoria inmunológica estarían más deprimidos ${ }^{35}$.

Finalmente, podemos concluir que en la Región Metropolitana la diabetes mellitus tipo 1 sigue manteniendo un patrón de crecimiento progresivo y que el análisis por comunas es concordante con aquellas localidades donde la población es eminentemente caucasoide, donde hay un mayor ingreso económico y un mayor estándar de urbanización. Nuestro grupo se encuentra trabajando en la aplicación de modelos Bayesianos que nos permitan aclarar si el aumento en las tasas de incidencia de diabetes tipo 1 en la Región Metropolitana, obedece a un patrón de agrupación témporo-espacial específico.

7. CRUz-Coke R. Origen y evolución étnica de la población chilena (Ethnic origin and evolution of the Chilean population). Rev Méd Chile 1976; 104: 365-8.

8. Censo 2002. Instituto Nacional de Estadísticas (INE). Resultados generales. Censo de Población y Vivienda 2002. Santiago: INE, 2004.

9. Larenas G, Montecinos A, Manosalva A, Barthou $\mathrm{M}, \mathrm{VIDAL}$ T. Incidence of insulin-dependent diabetes mellitus in the IX region of Chile: ethnic differences. Diab Res Clin Pract 1996; 34 Suppl: 147-51.

10. Carrasco E, Pérez-Bravo F, Santos JL, López G, Calvilíán M, WolfF $C$ et al. One of the lowest validated incidence of insulin dependent diabetes mellitus in the Americas: Santiago, Chile. Diab Res Clin Pract 1996; 34: 153-7.

11. Gondolf EW, Chang YF, Laporte R. Capture-recapture analysis of batterer reassaults: an epidemiological innovation for batterer program evaluation. Violence and Victims 1999; 14: 191-202. 
12. MuRRAy CJL, López AD. Global burden of disease and injury series. Volume II. Global health statistics. A compendium of incidence, prevalence and mortality estimates for over 200 conditions. Boston, Harvard School of Public Health on behalf the World Health Organization and the World Bank, 1996; 140.

13. Hyppönen E, Kenward MG, Virtaren SM, Pitulainen A. The childhood Diabetes in Finland (DiMe) study group. Infant feeding, early weight gain risk of type 1 diabetes. Diabetes Care 1999; 22: 1261-5.

14. Codner E, Mericq V, García H, López C, Cáceres J, Gaete X et aL. Resultados de un programa multidisciplinario de tratamiento intensificado de la Diabetes Mellitus tipo 1 (DM1) en un Hospital Público. Rev Méd Chile 2003; 131: 857-64.

15. Pérez-Bravo F, Oyarzún A, Carrasco E, Albala C, Dorman J, SANTOS JL. Duration of breast feeding and bovine serum albumin antibody levels in type 1 diabetes: a case-control study. Pediatric Diab 2003; 4: 157-61.

16. Carrasco E, Pérez-Bravo F, Dorman JS, Mondragón A, SANTos JL. Increasing incidence of type 1 diabetes in population from Santiago of Chile: Trends in a period of 18 years (1986-2003). Diab Metab Res Rev 2005 (on line, May 2005).

17. Casu A, Pascutto C, Bernasdinew L, Songin M. Type 1 diabetes among Sardinian children is increasing: The Sardinian diabetes register for children aged 0-14 years (1989-1999). Diabetes Care 2004; 27: 1623-9.

18. CAmpbei-Stokes PL, Tay Lor BJ. Prospective incidence study of diabetes mellitus in New Zealand children aged 0 to 14 years. Diabetologia 2005; 48: 643-8.

19. Newhook LA, Curtis J, Hagerty D, Grant M, Paterson AD, CRUMmel $C$ et al. High incidence of childhood type 1 diabetes in the Avalon peninsula, Newfoundland, Canada. Diabetes Care 2004; 27: 885-8.

20. Permutt MA, Wasson J, Cox N. Genetic epidemiology of diabetes. J Clin Invest 2005; 115: 1431-9.

21. Hyppönen E, ViRTANen SM, Kenward MG, KNip M, AKERBLOM HK. Obesity, increased linear growth, and risk of type 1 diabetes in children. Diabetes Care 2000; 23: 1755-60.

22. EURODIAS Substudy 2 Study Group. Rapid early growth is associated with increased risk of childhood type 1 diabetes in various European populations. Diabetes Care 2002; 25: 1755-60.

23. Karvonen M, PitKaniemi J, Tuomiento J. The onset age of type 1 diabetes in Finnish children has become younger. The Finnish Childhood Diabetes Registry Group. Diabetes Care 1999; 22: 1066-70.
24. Gardner SG, Bingley PJ, Sawtell PA, Weeks S, Gale EA. Rising incidence of insulin dependent diabetes in children aged under 5 years in the Oxford region: time trend analysis. The Bart's-Oxford Study Group. Br Med J 1997; 315: 713-7.

25. Schoenle EJ, Lang-Murtiano M, Gschwend S, LeimbaCHER J, Mu山s PE, Torresani T et al. Epidemiology of type I diabetes mellitus in Switzerland: steep rise in incidence in under 5 year old children in the past decade. Diabetologia 2001; 44: 286-9.

26. Kibirige M, Metcalf B, RenUKa R, WiLKin TJ. Testing the accelerator hypothesis: the relationship between body mass and age at diagnosis of type 1 diabetes. Diabetes Care 2003; 26: 2865-70.

27. Libman iM, Pietropaolo M, Arslanian SA, LaPorte $\mathrm{RE}, \mathrm{BECKER}$ DJ. Changing prevalence of overweight children and adolescents at onset of insulintreated diabetes. Diabetes Care 2003; 26: 2871-5.

28. RosenBLOOM AL Obesity, insulin resistance, betacell autoimmunity, and the changing clinical epidemiology of childhood diabetes. Diabetes Care 2003; 26: 2954-6.

29. Todaro M, Zeuner A, Stassi G. Role of apoptosis in autoimmunity. J Clin Immunology 2004; 24: 1-11.

30. Stene LC, Magnus P, Le RT, Sovik O, Joner G. Matemal and patemal age at delivery, birth order, and risk of childhood onset type 1 diabetes: population based cohort study. Br Med J 2001; 323: 369-71.

31. Sumnik Z, Drevinek P, Lanska V, Malcoma H, Vavrinec $\mathrm{J}$, CINEK O. Higher maternal age at delivery, and lower birth orders are associated with increased risk of childhood type 1 diabetes mellitus. Exp Clin Endocrinol Metab 2004; 112: 294-7.

32. Rautava S, Ruuskanen O, Ouwehand A, Salminen S, Isolauri $\mathrm{E}$. The hygiene hypothesis of atopic disease- an extended version. J Pediatr Gastroenterol Nutr 2004; 38: 378-88.

33. Estadísticas Sociales de los Pueblos Indígenas en Chile. Censo 2002. INE (2005), pp 117-8.

34. Encuesta CASEN (2000). http://www.mideplan.cl

35. Christen U, von Herat MG. Infections and autoimmunity-good or bad?J Immunol 2005; 174: 7481-6.

\section{Agradecimientos}

Los autores agradecen la colaboración de la Fundación de Diabetes Juvenil de Chile (Sra. Carolina Kaller y Sr. César Velasco). Esta investigación ha sido financiada gracias a los proyectos de investigación: DI Multidiciplinario MULT 04/17-2 (Prof. Elena Carrasco) y Fondecyt 1030680 (Prof. Francisco Pérez). 